\title{
The Impact of Fatigue on Patients with Multiple Sclerosis
}

\author{
John D. Fisk, Amanda Pontefract, Paul G. Ritvo, Catherine J. Archibald \\ and T.J. Murray
}

\begin{abstract}
Although fatigue is recognized as a symptom of MS, there have been insufficient methods for evaluating this symptom. We administered the Fatigue Impact Scale to 85 MS patients and 20 hypertensive patients. Neurologic impairment, mental health, and general health status were also assessed. MS patients reported significantly higher fatigue impact than hypertensive patients. Most MS patients reported fatigue as either their worst (14\%), or one of their worst (55\%) symptoms. Disease classification and neurologic impairment had little bearing on Fatigue Impact Scale scores in the MS sample. The best predictive models for mental health and general health status in the MS sample both included the Fatigue Impact Scale as a significant factor. This study demonstrates that: 1) fatigue is a very prevalent and severe problem in MS, 2) fatigue impact cannot be predicted by clinical measures of neurologic impairment, 3) fatigue has a significant effect on the mental health and general health status of MS patients.
\end{abstract}

\begin{abstract}
Résumé: L'impact de la fatigue chez les patients atteints de sclérose en plaques. Bien qu'il soit reconnu que la fatigue est un des symptômes de la sclérose en plaques, il n'existe pas de méthode satisfaisante pour évaluer ce symtôme. 85 patients souffrant de la sclérose en plaques et à 20 patients souffrant d'hypertension ont été évalués au moyen de l'échelle de l'impact de la fatigue (Fatigue Impact Scale). Nous avons également évalué la déterioration neurologique, la santé mentale et la santé en général. Les patients souffrant de la sclérose en plaques ont indiqué que l'impact de la fatigue était supérieur de façon significative à celui décrit par les patients souffrant d'hypertension. La majorité des patients souffrant de la sclérose en plaques ont indiqué que la fatigue était soit leur pire problème (14\%), soit l'un des plus sévères $(55 \%)$. La classification de la maladie ainsi que la sévérité de la détérioration neurologique étaient peu reliées à la fatigue, telle qu'évaluée par l'échelle de l'impact de la fatigue, chez les patients souffrant de la sclérose en plaques. Les résultats du l'échelle de l'impact de la fatigue constituaient un facteur significatif dans l'élaboration de modèles prédisant le mieux la santé mentale et la santé en général. Cette étude démontre que: 1) la fatigue est un problème fréquent et grave dont souffrent ceux qui sont atteints de la sclérose en plaques, 2) l'impact de la fatigue ne peut être prédit par les mesures de la détérioration neurologique, 3) la fatigue a un effet significatif sur la santé mentale et sur la santé en général des patients souffrant de la sclérose en plaques.
\end{abstract}

Can. J. Neurol. Sci. 1994; 21: 9-14

Fatigue is recognized as a disabling symptom of Multiple Sclerosis (MS) but measurement of this symptom has proven difficult and ratings of fatigue are typically not included in the routine quantitative evaluation of neurologic impairment." Although limited in number, studies that have addressed the issue of fatigue in MS patients have found fatigue to be of profound significance for patients. A previous survey of patients attending the Dalhousie MS Research Unit found that $76 \%$ of patients felt that the degree and type of fatigue that they experienced differed following the onset of the disease. ${ }^{2}$ Furthermore, $27 \%$ of these patients reported fatigue as a persistent symptom while $40 \%$ listed fatigue as their most serious symptom. Studies from other centers have yielded similar data. Krupp and colleagues ${ }^{3}$ reported that $28 \%$ of their sample of MS patients indicated that fatigue was their "most troubling symptom". Responses to a mail-out survey conducted by Freal et al. ${ }^{4}$ revealed that $48 \%$ of their sample of MS patients felt that fatigue resulted in a worsening of other symptoms. Studies such as these and awareness of the importance of fatigue among clinicians, have led to clinical trials aimed at ameliorating this symptom. ${ }^{2,5,6}$ Regardless of the recent interest in fatigue as a symptom of MS, there are relatively few instruments with established psychometric properties that can be used to measure the MS patient's subjective experience of this symptom. Perhaps because of this, it has proven difficult to find physiologic measures that have a clear relationship to subjective measures of fatigue. $^{7}$

From the Department of Psychology, Camp Hill Medical Centre (J.D.F., P.G.R.); Department of Psychiatry (J.D.F., A.P., P.G.R.), Department of Psychology (J.D.G., P.G.R., G.J.A.), Department of Medicine (J.D.F., T.J.M.), Dalhousie University. RECEIVED FEBRUARY 25, 1993, ACCEPTED IN FINAL FORM AUGUST I I, 1993.

Reprint requests to: John D. Fisk, Ph.D., Department of Psychology, Camp Hill Medical Centre, 1763 Robie Street, Halifax, Nova Scotia, Canada B3H 3G2 
Krupp et al. ${ }^{8}$ have reported a measure of fatigue that differentiates MS patients and patients with systemic lupus erythematosus from normal controls. However, this "fatigue severity scale" generates a single summary score from questions addressing very disparate aspects of the patient's experience of fatigue. For example, general statements, such as being "easily fatigued" and fatigue causing "frequent problems", are combined with very specific questions regarding the relationship between fatigue and motivation and fatigue and exercise. Thus, although this scale differentiates patients who have problems due to fatigue from those who do not, it has limited utility for examining the ways in which fatigue affects patients' lives. In order to improve our understanding of the effects of fatigue on the lives of patients with chronic disease, we developed a more detailed measure that is designed to assess the problems in patients' quality of life that they attribute to their symptoms of fatigue. This measure was designed as a specific health status measure according to the taxonomy put forth by Guyatt et al. ${ }^{9}$ to describe health-related quality of life measures.

In a previous investigation we found that ratings of fatigue correlated significantly with self-reported mental health in a sample of 105 MS patients. ${ }^{10}$ However, this study used only a simple rating of current fatigue severity, derived from a subscale of the Profile of Mood States"1. A methodological problem in this previous study ${ }^{10}$ was the potential overlap between measures of fatigue and measures of mood. Fatigue is considered symptomatic of mood disorders, ${ }^{12}$ and since studies of MS patients have reported high prevalence rates for both fatigue ${ }^{2.3}$ and mood disorders, ${ }^{13}$ measurement of either of these symptoms in isolation is problematic in studies of MS.

\section{Methods}

\section{Subjects}

The study sample included 85 patients ( 14 males, 71 females) attending the Dalhousie University MS Research Unit with a diagnosis of clinically probable (13) or clinically definite (72) MS. ${ }^{14}$ The patients ranged in age from 19 to 71 years (mean $=42.2, \mathrm{SD}=11.4$ ) and had a self-reported duration of MS symptoms from 1 to 49 years (mean $=12.7, \mathrm{SD}=9.4$ ). All information was collected within 8 weeks of a clinical assessment at which time ratings of neurologic impairment were completed by the neurologists or the nurse specialist of the Research Unit, using the Expanded Disability Status Scale (EDSS). ${ }^{1}$ EDSS scores of the MS sample ranged from 0 to 8.5 (mean = $3.9, \mathrm{SD}=2.1$ ). On the basis of current and past clinical examinations, the patients were classified into benign $(n=6)$, relapsing-remitting $(n=36)$, relapsing-progressive $(n=17)$, and chronic-progressive $(n=26)$ subgroups. Five of the patients reported taking antidepressant medications at the time of testing.

A comparison group of hypertensive clinic-attending patients was also examined. This group was selected in order to provide a baseline measure for the reporting of fatigue symptoms by patients without neurologic disease or primary complaints of fatigue. Attendance at a specialty medical clinic was felt to be a factor that could influence the likelihood that patients would acknowledge problems in everyday functioning and would attribute them to their symptoms. Therefore, the comparison group of hypertensive (HT) patients included 20 patients (10 males, 10 females) with mild to moderate hypertension who attended the Hypertensive Unit of Camp Hill Medical Centre.
All of these patients were receiving psychological services in the form of individual or group treatment programs for behavioral management of blood pressure. Diastolic blood pressure readings obtained on regular clinic visits ranged from 90 to 100 $\mathrm{mm}$ for this group. They ranged in age from 27 to 67 years (mean $=46.0, \mathrm{SD}=9.2$ ) and none had a history of past or current neurologic disease or psychiatric illness.

\section{Instruments and Procedure}

The new measure of fatigue employed in the current study was designed in order to allow us to evaluate the perceived impact of fatigue on the lives of MS patients, the factors that affect patients' perceptions of fatigue impact, and how fatigue may affect the mental health and general health status of MS patients. In constructing the Fatigue Impact Scale (FIS), we adopted the viewpoint expressed by the Canadian MS Research Group 5 that "measuring the effect of fatigue on activities ... is more sensitive than simply asking patients to rate fatigue" (pg. 277-8). A similar perspective has been presented by Monks, ${ }^{15}$ who emphasized that symptoms must be considered in terms of the patient's own expected activity levels and relationships, and compared to those situations in which these expectations are not met.

Items for the FIS were selected on the basis of existing fatigue questionnaires ${ }^{3,4}$ and interviews with $30 \mathrm{MS}$ patients from our previous study in which they described the impact of fatigue on their lives. These interviews were recorded on audiotape and the patients' statements were transcribed and sorted by the investigators (J.D.F., P.G.R., C.J.A.). The FIS was constructed to include three subscales to assess perceived fatigue impact on cognitive functioning ( 10 items), physical functioning (10 items), and psychosocial functioning ( 20 items) (see Appendix A). The grouping of the items into subscales reflected the interview responses of our MS patients as well as dimensions established in other health status and "quality of life" assessment instruments. ${ }^{16,17}$ In the FIS, the patients are asked to rate the extent that fatigue has caused problems for them in relation to exemplar statements $(0=$ No Problem to $4=$ Extreme Problem, Maximum FIS score $=160$ ). A grade 8 reading level is sufficient for reading the content of the questionnaire. Additional descriptive data regarding the frequency and duration of fatigue symptoms and conditions that worsen or reduce fatigue symptoms are obtained through questions that precede the 40 FIS items.

In addition to the FIS, all patients completed a number of other self-report assessment instruments. Mental health and general health status were considered to be primary outcome variables that could be affected by fatigue. These were evaluated with the Mental Health Inventory $(\mathrm{MHI})^{18}$ and the Sickness Impact Profile (SIP), ${ }^{19}$ respectively. The MHI was chosen as a measure of mental health since it provides a valid measure of mood which evaluates both positive well-being as well as psychological distress and since it does not include items reflecting subjective fatigue when evaluating impaired mood. The SIP was considered an important outcome variable to include in this study because it assesses the effects of MS on patients' lives in terms of standard behavioral indices. It provides a measure of general health status that is based on the patient's description of how their functioning has been limited by their disease. The SIP also has been used with a variety of diagnostic groups ${ }^{20-24}$ 
and is considered to be a valid, broad-based measure of health status. ${ }^{24}$ Since the content of two of the SIP subscales ("Sleep and Rest", "Alertness Behaviour") overlap in content with the FIS, however, the SIP total score that was used for all analyses was calculated with these overlapping items excluded. The potential contribution of a number of other factors to mental health and general health status was also assessed. The presence and significance of stressful life events was assessed with the Social Readjustment Rating Scale (SRRQ) ${ }^{25}$ while the perceived quality of social relationships, was assessed with the Revised UCLA Loneliness Scale (R-UCLA). ${ }^{26}$ Lastly, patients were asked to identify the duration of time that they had experienced symptoms of MS. This was judged to be the most accurate estimate of the time period that the patients had had to adjust to their MS symptoms.

All measures were administered by research staff using interview and written self-report formats in a single session with the patient. Administration of the measures took place either within the medical center or, in privacy, within the patient's home or local community. The study was approved by the Research Ethics Committee of Camp Hill Medical Centre and all patients provided written informed consent prior to administration of the measures.

\section{RESULTS}

The responses of the MS and HT groups to the FIS, MHI and SIP were compared using multivariate analyses of variance. Significant group differences were evident in the overall analyses (Wilk's Lambda $=.68, \mathrm{p}<.001$ ). Univariate comparisons revealed no significant differences for the comparison of $\mathrm{MHI}$ total scores $(F=2.53, p>.10)$ between the MS (mean $=168.9$, sd $=29.3$ ) and HT (mean $=157.7$, sd $=23.42$ ) groups. However, a significant difference $(F=18.37, p<.001)$ was found between the SIP total scores of the MS (mean $=16.97$, sd $=11.09$ ) and $\mathrm{HT}$ (mean $=5.87$, sd $=6.71$ ) groups. In addition, the MS group reported significantly greater problems associated with fatigue symptoms (mean $=66.8$, sd $=36.0$ ) than the HT group $($ mean $=29.2, \mathrm{sd}=29.6$ ) when the FIS total score was examined $(F=18.78, p<.001)$. Examination of the Cognitive, Physical and Psychosocial subscale scores of the FIS with separate multivariate analyses of variance revealed a significant overall effect of group (Wilk's Lambda $=.80, p<.001$ ) as well

Table 1. Comparison of mean (standard deviation) of health status measure scores for Multiple Sclerosis (MS) and Hypertensive (HT) Patients.

\begin{tabular}{lrr}
\hline & MS & HT \\
\hline Mental Heath Inventory & $168.9(29.3)$ & $157.7(23.4)$ \\
Sickness Impact Profile & $17.0(11.1)$ & $5.9(6.7)^{*}$ \\
Fatigue Impact Scale & $66.8(36.0)$ & $29.2(29.6)^{*}$ \\
\hline
\end{tabular}

FIS Subscale Scores (as percent of maximum score)

\begin{tabular}{lll} 
Cognitive & $36.2(24.0)$ & $18.1(18.2)^{*}$ \\
Physical & $49.9(26.1)$ & $19.0(20.7)^{*}$ \\
Psychosocial & $40.4(22.3)$ & $17.9(19.1)^{*}$ \\
\hline
\end{tabular}

* Univariate $F>9.9, \mathrm{p}<.005$ as significant differences on all three subscale scores ( $\mathrm{F}>9.90$, $\mathrm{p}<.005$ for all comparisons) (see Table 1). Examination of the internal consistency of the FIS and its subscales revealed very high internal consistency in the responses by both groups (Cronbach's alpha $>.93$ for all analyses).

Sixty-nine percent of the MS patients reported that fatigue was either their worst symptom (12/85) or one of their worst symptoms (47/85). Only eight percent (7/85) reported that they did not experience problems due to fatigue. The remaining MS patients reported fatigue to be either a less severe (11/85), or their least severe $(8 / 85)$ symptom. Sixty percent reported that fatigue made their other symptoms worse. The majority of MS patients $(71 / 85)$ reported that they frequently or always became

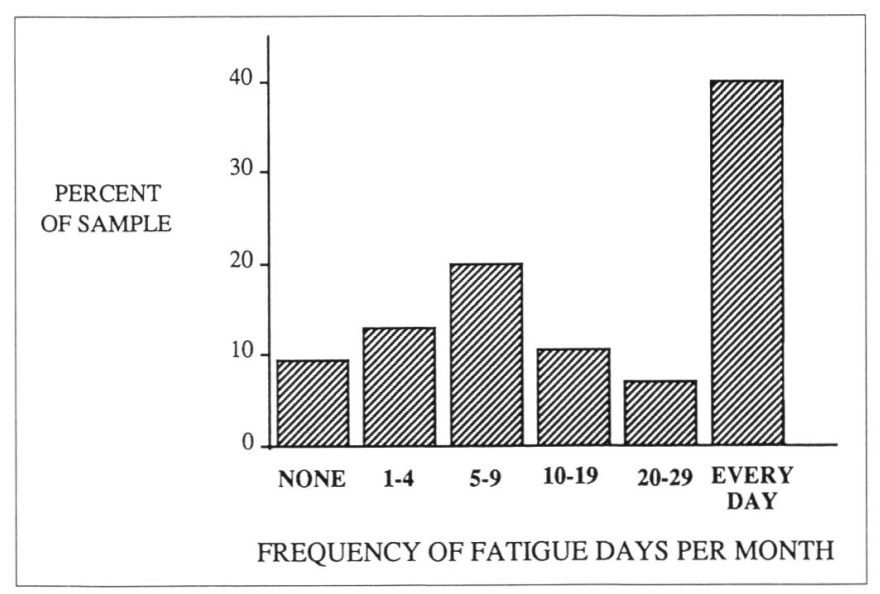

Figure 1: Frequency of fatigue problems during the past 30 days for the $M S$ patients.

fatigued when exposed to increased temperatures. Approximately one-half (43/85) of the MS patients reported that they had noticed problems due to fatigue before they had noticed other symptoms of MS. Correlational analysis with typical clinical information such as EDSS scores $(r=.065, p=$ $.552)$, patient age $(r=.059, p=.590)$, and self-reported duration of MS symptoms $(r=-.062, p=.569)$, failed to show any relationship with fatigue impact as measured by the FIS.

The estimated frequency with which fatigue had been a problem for the MS patients in the past 30 days is illustrated in Figure 1. Forty percent of the MS patients (34/85) reported problems due to fatigue every day. The patients were also asked to estimate the usual duration of fatigue symptoms when they occurred. In our MS sample, approximately $44 \%$ (37/85) reported a typical duration of less than 6 hours, and approximately equal proportions of patients reported average durations from 6 to 12 hours (19/85) and from 12 to 24 hours (22/85).

In order to examine factors which could be related to the impact of fatigue, the FIS subscale scores were examined via multivariate analysis of variance (SAS, GLM Procedure) with fatigue frequency, duration of fatigue symptoms, and MS classification (benign, relapsing-remitting, relapsing-progressive, chronic-progressive) as group variables. Significant main effects were found in the multivariate analysis of fatigue frequency (Wilk's Lambda $=.60, p<.002$ ) and MS classification (Wilk's Lambda $=.73, p<.01$ ) but not for the usual duration of fatigue symptoms (Wilk's Lambda $=.86, p>.05$ ). No 


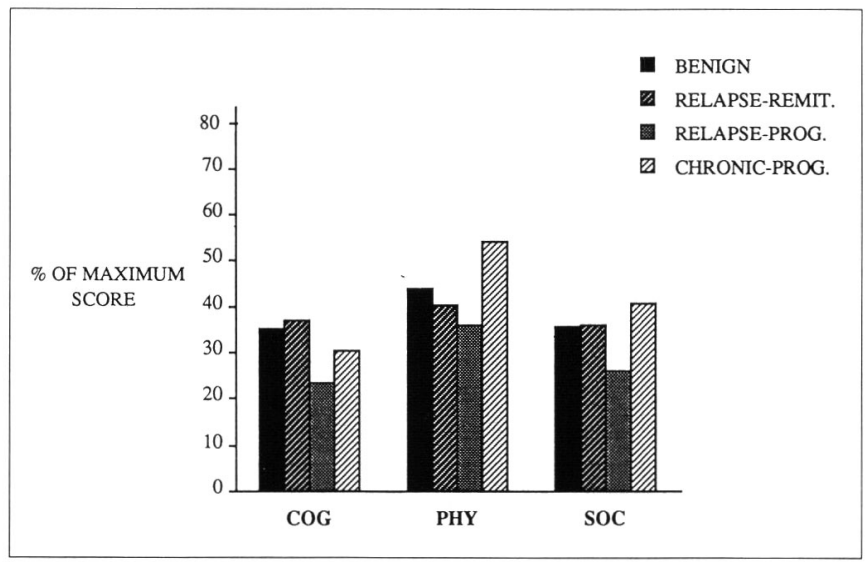

Figure 2: Least-squares adjusted means on the Cognitive (COG), Physical (PHY) and Psychosocial (SOC) subscales scores (expressed as percent of maximum score) for subgroups of the MS sample.

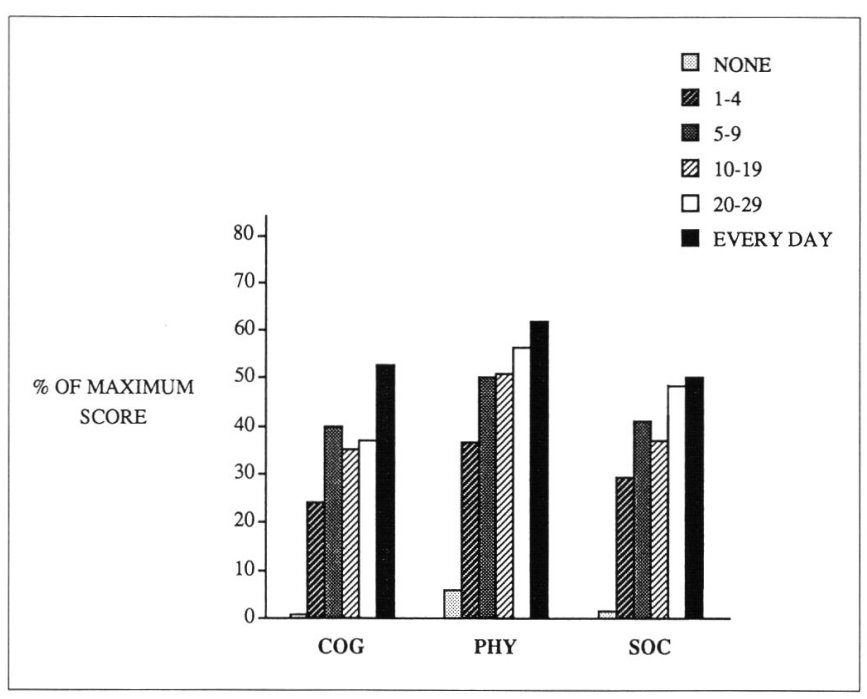

Figure 3: Least-squares adjusted means on the Cognitive (COG), Physical (PHY) and Psychosocial (SOC) subscales scores (expressed as percent of maximum score) for MS patients grouped according to the frequency of fatigue symptoms.

significant interactions were found between group variables. The overall effect of MS subgroups on FIS subscale scores reflected the relatively greater impact of fatigue on physical functioning in Chronic Progressive patients. These patients demonstrated significantly higher physical subscale scores than either relapsing-remitting or relapsing-progressive patients (pairwise comparisons $p<.01$ ). No other pairwise comparisons reached statistical significance (see Figure 2). The overall effect of fatigue frequency was primarily due to the fact that significantly greater impact was reported by those patients who experienced fatigue every day. Patients who reported daily fatigue demonstrated significantly higher FIS subscale scores than patients who reported either no fatigue or fatigue on 1-4 days, 59 days or $10-19$ days in the past month $(p<.05)$. No other pairwise comparisons reached statistical significance (see Figure 3 ).

The relationships between the self-reported effects of fatigue and measures of mental health and general health status were examined via stepwise multiple regression analyses with the
MHI and the SIP as outcome measures. The independent variables for both analyses included: age, duration of symptoms, EDSS score, SRRQ score, R-UCLA score, and FIS total score. A forward selection procedure was used with the probability of $F$ to enter set to $p<.05$. Only the FIS score was included in the regression model for both outcome measures. The best predictive model for mental health included fatigue impact (FIS), perceived quality of social relationships (R-UCLA) and the duration of MS symptoms. Together, these measures accounted for $68 \%$ of the variance in MHI scores. When the FIS score was entered first into this model, it alone accounted for $38 \%$ of the variance in MHI scores. The best predictive model for general health status (SIP) once again included fatigue impact (FIS) and also included neurologic impairment (EDSS) and the presence and significance of stressful life events (SRRQ). Together, these measures accounted for $66 \%$ of the variance in SIP scores. The EDSS entered first into this model and accounted for $35 \%$ of the variance in SIP scores. The FIS entered second and accounted for an additional $26 \%$ of the SIP variance.

\section{Discussion}

This study supports the findings of previous studies of fatigue which have pointed out the high prevalence and subjective severity of fatigue in MS patients. ${ }^{2-4}$ The impact of fatigue was clearly higher for the MS patients than for a clinic-attending sample of hypertensive patients and the responses to the FIS items by both groups revealed a high degree of internal consistency for this measure. Of particular importance is the finding that despite the significant differences between these two groups in their experience of fatigue, no such difference was found in their self-reported mental health status. Thus, it cannot be argued that the increased impact of fatigue reported by our sample of MS patients is caused by relatively depressed mood in this group of patients. Based on these findings, the FIS appears to be an appropriate self-report instrument for assessing the subjective experience of fatigue by MS patients.

Typical clinical factors had little bearing on the impact of fatigue that was reported by the MS patients. This was reflected in the absence of any significant correlation between FIS scores and either the time since self-reported onset of MS symptoms or the current EDSS scores. This inability to predict the severity of fatigue on the basis of routine clinical assessment (i.e., EDSS scores) appears to be a robust finding since others have reported similar findings with differing methods of assessing fatigue. ${ }^{3}$ Disease classification did affect the reported impact of fatigue, but only the impact on physical functioning. Although significantly higher physical fatigue scores were reported by the chronic-progressive group, disease classification had no effect on the patients' reports of the impact of fatigue on cognitive and psychosocial functioning (see Figure 2). The high level of fatigue impact reported by patients with benign MS was quite noteworthy. Given the small number of patients in this group (i.e., 6), this finding may represent sampling bias. However, it is also possible that in the absence of progressively worsening or variable neurologic symptoms, fatigue is the most salient and functionally limiting symptom for benign MS patients. Further study of a larger group of such patients is clearly warranted.

The overall impact of fatigue was determined primarily by how often fatigue was experienced rather than by disease classification or the usual length of time per day that patients were 
fatigued. The most profound impact of fatigue was seen in those patients who reported that they had experienced problems due to fatigue on each of the past 30 days. This included almost half of the MS patient sample. It was this group in particular, who reported that fatigue adversely affected their perceptions of their cognitive functioning (see Figure 3). Given that almost half of the MS patients reported fatigue every day, and given that the impact of fatigue was related to its frequency, it is not surprising that over half of our MS patient sample reported fatigue to be either their worst, or one of their worst symptoms.

Our findings suggest that the disruption of MS patients' lives that results from their need to accommodate fatigue or its potential occurrence, adversely affects their mental health. This important relationship between fatigue and mental health, which was suggested by our previous research, ${ }^{10}$ and which was confirmed in the present study, has not been examined in other studies of fatigue in MS. The strong relationship between fatigue and mental health is an important result which stands in clear contrast to the lack of a significant relationship between clinical ratings of neurologic impairment and mental health. Despite the lack of significant differences in MHI scores between the MS and HT groups in the present study, it is important to note that the mean scores for both groups fell in the lowest third of the distribution of scores obtained from a normative population in the original Rand Corporation study with this measure. ${ }^{27}$ Thus, it can be argued that both of our clinic samples demonstrated evidence of relatively poor mental health. Since previous studies have shown MHI scores to have value in predicting the future use of mental health services, ${ }^{28}$ careful monitoring of the MS patient's fatigue problems may alert the clinician to look for significant mental health problems that might not otherwise be disclosed in the clinical assessment.

The present study demonstrated that fatigue has a strong relationship not only with mental health, but also with more broadbased measures of general health status. FIS scores alone accounted for a substantial proportion of the variance in SIP scores and contributed a unique component to this model of general health status, in addition to neurological impairment. These findings indicate that fatigue must be considered an important predictor of the extent to which the MS patient's general health is affected by the disease. Since $60 \%$ of our sample reported a worsening of other symptoms when fatigued, assessment of fatigue has obvious importance in the routine clinical examination. Determining how a patient may be affected by fatigue and assessing their present experience of fatigue is essential to understanding the extent to which the patient's presentation in the clinical examination reflects their general health status. Assessments of neurologic impairment and neurologic symptoms without an assessment of the impact of fatigue would appear to be insufficient information to allow the clinician to accurately estimate the MS patient's health status since this ignores a symptom which was rated as one of the most significant complaints by approximately two-thirds of our patient sample. Fatigue appears to be a distinct impairment factor which, together with neurologic impairment, plays a large role in determining the physical, psychological and social handicaps that the MS patient faces.

\section{APPENDIX}

The Fatigue Impact Scale asks patients to rate how much of a problem fatigue has caused them during the past month, including the day of testing in reference to the statements listed below. The patient is asked to circle the appropriate response for each: 0 - No Problem; 1 - Small Problem; 2 - Moderate Problem; 3 - Big Problem; 4 - Extreme Problem. The item number in brackets following each statement indicates the order in which it is presented in the Fatigue Impact Scale.

\section{Cognitive Dimension}

Because of my fatigue:

- I feel less alert. [1]

- I have difficulty paying attention for a long period of time. [5]

- I feel like I cannot think clearly. [6]

- I find that I am more forgetful. [11]

- I find it difficult to make decisions. [18]

- I am less motivated to do anything that requires thinking. [21]

- I am less able to finish tasks that require thinking. [26]

- I find it difficult to organize my thoughts when I am doing things at home or at work. [30]

- I feel slowed down in my thinking. [34]

- I find it hard to concentrate. [35]

\section{Physical Dimension}

Because of my fatigue:

- I am more clumsy and uncoordinated. [10]

- I have to be careful about pacing my physical activities. [13]

- I am less motivated to do anything that requires physical effort. [14]

- I have trouble maintaining physical effort for long periods. [17]

- My muscles feel much weaker than they should. [23]

- My physical discomfort is increased. [24]

- I am less able to complete tasks that require physical effort. [31]

- I worry about how I look to other people. [32]

- I have to limit my physical activities. [37]

- I require more frequent or longer periods of rest. [38]

\section{Social Dimension}

Because of my fatigue:

- I feel that I am more isolated from social contact. [2]

- I have to reduce my workload or responsibilities. [3]

- I am more moody. [4]

- I work less effectively. (This applies to work inside or outside the home.) [7]

- I have to rely more on others to help me or do things for me. [8]

- I am more irritable and more easily angered. [12]

- I am less motivated to engage in social activities. [15]

- I have few social contacts outside of my own home. [19]

- Normal day-to-day events are stressful for me. [20]

- I avoid situations that are stressful for me. [22]

- I have difficulty dealing with anything new. [25]

- I feel unable to meet the demands that people place on me. [27]

- I am less able to provide financial support for myself and my family. [28]

- I engage in less sexual activity. [29]

- I am less able to deal with emotional issues. [33]

- I have difficulty participating fully in family activities. [36]

- I am not able to provide as much emotional support to my family as I should. [39]

- Minor difficulties seem like major difficulties. [40]

- I have difficulty planning activities ahead of time because my fatigue may interfere with them. [9]

- Fatigue limits my ability to travel outside my home. [16]

\section{ACKNOWLEDGEMENTS}

This research was supported by The National Health Research and Development Program (Health and Welfare Canada), Project No. 66031344-MH (64/1).

We gratefully acknowledge the assistance of Dr. C.E. Maxner, Dr. V. Bhan and Pauline Weldon, R.N. of the MS Research Unit, as well as Debbie McLeod, R.N., Dr. C. Abbot, Dr. J. Gray, and Dr. C.R.T. Dean 
of the Hypertensive Unit for providing us with the patients for this study. We would also like to thank research assistants Linda Awalt and Glenda Sherwood, R.N. for their contributions, and Wade Blanchard, M.Sc. and Dr. C. Field of the Dalhousie University Department of Mathematics for statistical analyses and consultation.

\section{REFERENCES}

1. Kurtzke JF. Rating neurologic impairment in multiple sclerosis: an Expanded Disability Status Scale (EDSS). Neurology 1983; 33: 1444-1452.

2. Murray TJ. Amantadine therapy for fatigue in multiple sclerosis. Can J Neurol Sci 1985; 12: 251-254.

3. Krupp LB, Alvarez LA, LaRocca NG, Scheinberg LC. Fatigue in multiple sclerosis. Arch Neurol 1988; 45: 435-437.

4. Freal JE, Kraft GH, Coryell JK. Symptomatic fatigue in multiple sclerosis. Arch Phys Med Rehabil 1984; 65: 135-138.

5. The Canadian Research Group. A randomized controlled trial of amantadine in fatigue associated with multiple sclerosis. Can J Neurol Sci 1987; 14: 273-278.

6. Cohen RA, Fisher M. Amantadine treatment of fatigue associated with multiple sclerosis. Arch Neurol 1989; 46: 676-680.

7. Sandroni P, Walker C, Starr A. "Fatigue" in patients with multiple sclerosis. Arch Neurol 1992; 49: 517-524.

8. Krupp LB, LaRocca NG, Muir-Nab J, Steinberg AD. The Fatigue Severity Scale: application to patients with multiple sclerosis and systemic lupus erythematosus. Arch Neurol 1989; 46: 1121 1123.

9. Guyatt GH, Van Zanten SJOV, Feeny DH, Patrick DL. Measuring quality of life in clinical trials: a taxonomy and review. Can Med Assoc J 1989; 140: 1441-1448.

10. Ritvo PG, Fisk JD, Archibald CJ, et al. A model of mental health in patients with multiple sclerosis. Can Psychol 1992; 33(2a): 391.

11. McNair D, Lorr M, Droppleman L. EDITS Manual for the Profile of Mood States. San Diego, CA: Educational and Industrial Testing Service, 1971.

12. American Psychiatric Association. Diagnostic and Statistical Manual of Mental Disorders-Revised. Washington, DC: American Psychiatric Association, 1987.

13. Minden, SL, Schiffer, RB. Affective disorders in multiple sclerosis: review and recommendations for clinical research. Arch Neurol 1990; 47: 98-104.
14. Kurtzke JF. Multiple sclerosis: what's in a name? Neurology 1988; 38: 309-316.

15. Monks J. Experiencing symptoms in chronic illness: fatigue in multiple sclerosis. Int Disabil Stud 1989; 11: 78-83.

16. Pollard WE, Bobbitt RA, Bergner M, et al. The Sickness Impact Profile: reliability of a health status measure. Med Care 1976; 14: 146-155.

17. Read JL, Quinn R, Hoefer, MA. Measuring overall health: an evaluation of three important approaches. J Chron Dis 1987; 40: 7s$21 \mathrm{~s}$.

18. Veit $\mathrm{C}$, Ware $\mathbf{J} \mathrm{Jr}$. The structure of psychological distress and wellbeing in general populations. J Consult Clin Psychol 1983; 5I: 730-742.

19. Bergner M, Bobbitt RA, Carter WB, Gilson BS. The Sickness Impact Profile: development and final revision of a health status measure. Med Care 1981; 19: 787-805.

20. Busenbark KL, Nash J, Nash S, Hubble JP, Koller WC. Is essential tremor benign? Neurology 1991; 41: 1982-1983.

21. Follick MJ, Smith TW, Ahern DK. The Sickness Impact Profile: a global measure of disability in chronic low back pain. Pain 1985; 21: 67-76.

22. Klonoff PS, Snow WG, Costa LD. Quality of life in patients 2 to 4 years after closed head injury. Neurosurgery 1986; 19: 735-743.

23. Prigatano GP, Wright EC, Levin D. Quality of life and its predictors in patients with mild hypoxemia and chronic obstructive pulmonary disease. Arch Intern Med 1984; 144: 1613-1619.

24. Bell M, Bombardier C, Tugwell P. Measurement of functional status, quality of life, and utility in rheumatoid arthritis. Arthritis Rheum 1990; 33: 591-601.

25. Monroe SM. Assessment of life events: retrospective versus concurrent strategies. Arch Gen Psychiatry 1982; 39: 606-610.

26. Russell D, Peplau LA, Cutrona CE. The Revised UCLA Loneliness Scale: concurrent and discriminant validity evidence. J Pers Soc Psychol 1980; 39: 472-480.

27. Davies AR, Sherbourne CD, Peterson JR, Ware JE. Scoring Manual: Adult Health Status and Patient Satisfaction Measures Used In RAND Health Insurance Experiment. Bethesda, MD: Department of Health and Human Services, 1988.

28. Ware JE, Manning WG Jr, Duan N, Wells KB, Newhouse JP. Health status and the use of outpatient mental health services. Am Psychol 1984; 39: 1090-1 100. 